
\title{
Besearch S Surare \\ Elevated coinhibitory molecule TIGIT mediates T cell exhaustion in septic patients
}

\section{Yini Sun}

China Medical University https://orcid.org/0000-0003-4399-137X

\section{Renyu Ding}

China Medical University

\section{Yukun Chang}

China Medical University

\section{Jiuming Li}

China Medical University

Xiaochun Ma ( $\sim$ maxc_cmu@sina.com )

China Medical University https://orcid.org/0000-0002-1714-9800

\section{Research}

Keywords: Sepsis, Septic shock, TIGIT, Anti-TIGIT Ab, T cell exhaustion

Posted Date: May 7th, 2021

DOI: https://doi.org/10.21203/rs.3.rs-475376/v1

License: (c) (1) This work is licensed under a Creative Commons Attribution 4.0 International License. Read Full License 


\section{Elevated coinhibitory molecule TIGIT mediates T cell exhaustion in septic patients}

Yini Sun, Renyu Ding, Yukun Chang, Jiuming Li and Xiaochun Ma*

Department of Critical Care Medicine, The First Affiliated Hospital of China Medical University, China Medical University, Shenyang, China

* Correspondence:

Xiaochun Ma, Ph.D.

maxc_cmu@sina.com 


\begin{abstract}
Background: Sepsis-induced T cell exhaustion that is characterized by upregulated coinhibitory molecules and decreased cytokines release plays a crucial role in the immunosuppression during sepsis. Although PD-1 has shown a promising target to interfere with T cells dysfunction, the role of other coinhibitory receptors in sepsis remains largely elusive. Recently, it has been demonstrated that the coinhibitory molecule TIGIT more reliably identified exhausted T cells than PD-1. The aim of the study was to identify the expression of TIGIT on lymphocytes and the crucial role of TIGIT in modulating $\mathrm{T}$ cell function in septic patients.
\end{abstract}

Methods: Twenty-five patients with sepsis and seventeen healthy controls were prospectively enrolled. Peripheral blood was obtained from septic patients within 24 hours after diagnosis of sepsis, as were healthy controls. TIGIT and other coinhibitory/costimulatory molecules expression on lymphocyte subsets was quantitated by flow cytometry. The relationship between TIGIT expression and clinical parameters was simultaneously evaluated. The function $\mathrm{T}$ cell from septic patients was assayed via stimulated cytokine secretion. Ex vivo functional assays were also conducted.

Results: In the early stage of sepsis, patients exhibited higher levels of TIGIT on T cells relative to healthy donors, especially in the septic shock patients. Elevated frequencies of $\mathrm{TIGIT}^{+} \mathrm{T}$ cells positively correlated with the severity of organ failure and inflammatory responses in septic patients. TIGIT $^{+} \mathrm{T}$ cells expressed higher levels of PD-1 and lower CD226. Further, elevated expression of TIGIT inhibited the release of cytokines including TNF, IFN- $\gamma$ and IL-2 by CD4 ${ }^{+}$ and $\mathrm{CD}^{+} \mathrm{T}$ cells. Strikingly, ex vivo blockade of TIGIT using anti-TIGIT antibody restored the frequencies of cytokine-producing $\mathrm{T}$ cells.

Conclusions: These data illustrate TIGIT as a novel marker of exhausted T cells and suggest TIGIT may be a novel immunotherapeutic target during sepsis.

Keywords: Sepsis, Septic shock, TIGIT, Anti-TIGIT Ab, T cell exhaustion 


\section{Background}

Sepsis is a dysregulated host response to infection and remains a major cause of mortality in the intensive care unit (1). Although the initial phase of sepsis has been historically considered to be due to over-inflammatory response, emerging evidence has demonstrated that disturbance of the balance of inflammatory and immune responses results in the high mortality of sepsis (2).

Sepsis-related immunosuppression contributes to a protracted course and poor outcome of the increased risk of nosocomial infection or even death $(3,4)$. The apoptosis, dysfunction and exhaustion of $\mathrm{T}$ cells contribute to sepsis-induced immunosuppression. An analysis of tissues obtained by autopsy from septic patients has identified $\mathrm{T}$ cell apoptosis and $\mathrm{T}$ cell exhaustion, which was characterized by decreased cytokine production and increased expression of coinhibitory receptors such as PD-1, CTLA-4 (5). Another prospective study demonstrated the expression of the inhibitory receptors CTLA-4, TIM-3 and LAG-3 on T lymphocytes was upregulated in the patients with severe sepsis (6). Targeting PD-1/PD-L1 has been reported to reverse T cell exhaustion in septic patients (7), and anti-PD-1 antibody has entered phase I clinical trials (8). Additionally, the blockade of CTLA-4 resulted in improved survival in the sepsis animal model (9). However, not all the features of the exhausted T-cells could be restored by interfering with single PD-1/PD-L1 or CTLA-4 pathway $(10,11)$. Therefore, new immune checkpoint and therapeutic approaches are needed to explore during sepsis.

TIGIT (T cell immunoglobin and ITIM domain) is a recently described immune checkpoint receptor that is mainly expressed on NK cells, $\mathrm{CD}^{+}$and $\mathrm{CD}^{+} \mathrm{T}$ cells and is upregulated upon activation on naïve $\mathrm{T}$ cells $(12,13)$. TIGIT competes with costimulatory receptor CD226 (also called DNAM) for the ligands CD155 expressed on dendritic cells and mediates immune suppression in tumors and chronic infections $(14,15)$. It has been reported that TIGIT is highly expressed on $\mathrm{T}$ cells in models of both cancer and viral infection of human and murine (16). A mice sepsis model study revealed that TIGIT expression on T cells was increased and CD155 blockade improved the survival by inhibiting TIGIT-CD155 interaction (17). Additionally, the disturbance of CD226 homodimerization by TIGIT can abrogate the costimulatory signals required for the activation of T cells (16). A more recent study indicated that while PD-1 expression had no differential at different stages of their differentiation, TIGIT expression was high on exhausted $\mathrm{T}$ 
cells but low on effector T cells (18). Thus, TIGIT expression could more reliably identify T cell exhaustion than PD-1.

To date, TIGIT expression and its effects on T cell function are still unknown during sepsis and septic shock. In this study, we interrogated the expression and function of TIGIT on lymphocytes from septic patients for the first time. Another goal of the study was to determine if interfering with the TIGIT pathway could reverse $\mathrm{T}$ cell exhaustion to gain insight into a possible target of immune intervention during sepsis. Our data demonstrated that compared with healthy donors, the TIGIT expression on $\mathrm{T}$ cells was higher in the septic patients, especially in the septic shock patients. Elevated expression of TIGIT positively correlated with inflammatory responses and ventilator time, and might be related to organ dysfunction and the occurrence of secondary nosocomial infection during sepsis. High expression of TIGIT suggested $\mathrm{T}$ cell exhaustion characterized by elevated PD-1, decreased CD226 and dampened cytokines production by T cells in sepsis. Strikingly, ex vivo antibody blockade of TIGIT reversed T cell exhaustion in septic patients. 


\section{Methods}

\section{Patients and controls}

This study was a prospective study performed in an ICU of a 3000-bed university-affiliated hospital in China. The study protocol was approved by the Research and Ethics Committee of the First Affiliated Hospital of China Medical University ([2015]2015-113-2, Shenyang, China), and written informed consent was obtained from each subject. Patients older than 18 years old admitted to the ICU who fulfilled the definition of sepsis 3.0 were included within the $24 \mathrm{~h}$ of diagnosis (1). Exclusion criteria included active malignancy, hematological or immunological diseases, pretreatment with immunosuppressive medications (except corticosteroids at a single dose of $<10 \mathrm{mg}$ prednisone or equivalent per day), and chronic viral infections (human immunodeficiency virus or hepatitis B or C). Control subjects consisted of healthy age and gender-matched volunteers.

\section{Data collection}

Baseline demographics included age, gender, comorbidity, APACHE II score, SOFA score, and the time from diagnosis to inclusion. Data were also collected regarding the source of infection, pathogens, the requirement for mechanical ventilator and continuous renal replacement therapy, ICU length of stay and ICU mortality. Septic patients were recorded and monitored daily for evidence of nosocomial infection until the discharge of hospital. Septic patients at admission of ICU have complete blood count analysis, procalcitonin (PCT) and C-reactive protein (CRP) performed in the clinical laboratory at the First Affiliated Hospital of China Medical University. The secondary nosocomial infection is defined as patients suffered from a new infection after the diagnosis of sepsis during the length of hospitalization.

\section{Sample collection and processing}

Within $24 \mathrm{~h}$ after the diagnosis of sepsis, $5.4 \mathrm{ml}$ blood sample was collected using an EDTA tube. The peripheral blood mononuclear cells (PBMCs) were isolated by Hypaque-Ficoll (GE life, USA) density gradient centrifugation and stored in liquid nitrogen. Plasma was collected and stored at $80^{\circ} \mathrm{C}$ for subsequent analysis. The levels of cytokines (IL-2, IL-4, IL-6, IL-10, IL-17, TNF, IFN- 
$\gamma)$ were quantified using the human Inflammatory cytokine Cytometric Bead Assay kit from BD (Canto II, USA) according to the manufacturer's recommendations.

\section{Flow cytometry}

The cryopreserved PBMCs were thawed and washed with PBS containing 2\% fetal bovine serum (FBS, Gibco). Viable Lymphocytes were identified by forward scatter (FSC) and side scatter (SSC) properties and exclusion of Live/Dead Fixable Aqua (ThermoFisher). T cell subsets were further identified by anti-CD3 (BD, SP34-2), anti-CD4 (BD, RPA-T4), and anti-CD8 (BD, RPAT8). Regulatory $\mathrm{T}$ cells were identified via intracellular staining for anti-Foxp3 (eBioscience, 236A/E7). NK cells were identified by anti-CD56 (Biolegend, 5.1H11). Also, anti-CD45RA (BD, HI100), anti-CCR7 (Invitrogen, 3D12), anti-TIGIT (Biolegend, A15153G), anti-PD-1 (Biolegend, EH12.2H7), anti-CD226 (Biolegend, TX25) were used for surface staining to determine T cell subsets and phenotype. For intracellular cytokine staining, PBMCs were stimulated with 50ng/ml phorbol myristate acetate (PMA, Sigma) and 1ug/ml ionomycin (Sigma) in the presence of Golgiplug (BD Pharmingen) for 4 hours at $37^{\circ} \mathrm{C}$, and then permeabilized and fixed using a BD Cytofix/Cytoperm kit following by staining with anti-TNF (Biolegend, MAb11), anti-IFN- $\gamma$ (Biolegend, 4S.B3), and anti-IL-2 (Biolegend, MQ1-17H12). Accucheck Counting Beads (Thermo Fisher Scientific) were added after staining to calculate the absolute number of lymphocytes. Samples were analyzed on an LSRII flow cytometer. Fluorescence minus one (FMO) samples were prepared to facilitate gating. Anti-mouse or anti-rat IgG-coated beads were stained with each fluorochrome-conjugated antibody and used for software-based compensation. Data were analyzed using FlowJo software (9.9.6 FlowJo, LLC).

\section{Ex vivo functional assays}

PBMCs were resuspended in culture medium (R10) consisting of RPMI 1640 containing 10\%

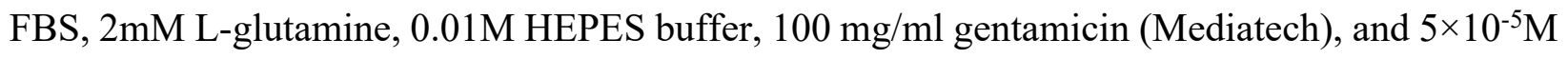
2-mercaptoethanol (Sigma-Aldrich, St.Louis, MO) at $1 \times 10^{6}$ cells each well of a round-bottom 96well tissue culture plate. The cells were either left unstimulated or treated with anti-CD3 (5 ug/ml. OKT3, eBioscience) and then treated with either $5 \mu \mathrm{g} / \mathrm{mL}$ anti-TIGIT antibody or isotype control antibody (eBioscience) for $3 \mathrm{~d}$ at $37^{\circ} \mathrm{C}$ in a $5 \% \mathrm{CO} 2$, humidified atmosphere. Some cultures were washed twice with media and re-stimulated with $50 \mathrm{ng} / \mathrm{mL}$ PMA and $1 \mu \mathrm{g} / \mathrm{mL}$ Ionomycin in the 
presence of Golgiplug for $5 \mathrm{~h}$ at $37^{\circ} \mathrm{C}$. Intracellular cytokine staining was performed after fixation and permeabilization using Foxp3 Staining Buffer Set (eBioscience) according to manufacturer's instructions, followed by staining with anti-Foxp3, anti-TNF, anti-IL-2 (Biolegend) and anti-IFN$\gamma$ as described above.

\section{Statistics}

Data shown and described depict the average \pm SEM. A two-tailed non-parametric Wilcoxon matched pairs test or a two-tailed Mann Whitney $U$ test was used for evaluation between two groups. One-way ANOVA with Turkey's multiple comparison tests was used to analyze data in more than two groups. Spearman's rank test was used for correlation analyses. All statistical analyses were conducted using GraphPad Prism 8.0 software (San Diego, CA). Two-tailed $p$ values $<0.05$ were considered statistically significant. 


\section{Results}

\section{Study cohort}

The clinical and laboratory values for septic patients, including age, gender, comorbidity, the scores of severity of illness, infection source, infection pathogens, length of ICU and ICU mortality et al. were provided in Table 1. The basic information of healthy donors (HD) was also listed in Table 1. The study enrolled a cohort of 25 septic patients, 15 of whom were diagnosed with septic shock and a cohort of 17 healthy donors. The mean age of the septic patients was 67.4 years old, with $76 \%$ male. The mean total white blood cell and lymphocyte counts were $13.5 \times 10^{9}$ cells $/ \mathrm{L}$ and $0.9 \times 10^{9}$ cells/L, respectively. The abdomen was the most common infection site (68\%), and Gramnegative bacteria were the most culprit pathogen (37\%) in the cohort of septic patients. All septic patients required mechanical ventilation, and $40 \%$ of septic patients suffered from a secondary nosocomial infection occurring during the hospitalization. The mortality in the septic and septic shock patients was $20 \%$ and $33 \%$, respectively (Table 1 ).

\section{TIGIT expression on $\mathbf{T}$ cells is upregulated during sepsis, especially in the patients with septic} shock

We assessed the expression of TIGIT on T and NK cells in the PBMCs from HD, sepsis and septic shock patients (Figure 1A, Supplementary Figure 1). Results showed TIGIT was significantly upregulated on bulk $\mathrm{CD} 4^{+}$and Foxp $3^{+} \mathrm{CD} 4^{+}$Treg cells in either septic or septic shock patients compared with HD (Figure 1B-1C). Nonetheless, there were no significant differences of TIGIT on Foxp3-CD4 ${ }^{+}$Tconv cells among HD, sepsis and septic shock patients (Figure 1D). Relative to $\mathrm{HD}$, the expression of TIGIT on bulk $\mathrm{CD}^{+} \mathrm{T}$ cells was upregulated in septic shock patients (Figure 1E). Additionally, there was no difference in TIGIT expression on NK cells among three groups (Figure 1F). Further, we assessed the TIGIT expression on the subpopulations of T cells and found that TIGIT expression was upregulated on naïve $\left(\mathrm{CD} 45 \mathrm{R}^{+} \mathrm{CCR} 7^{+}\right)$, effector memory $\left(\mathrm{CD} 45 \mathrm{RA}^{-}\right.$ $\left.\mathrm{CCR}^{-}\right)$and central memory $\left(\mathrm{CD} 45 \mathrm{R}^{-} \mathrm{CCR} 7^{+}\right) \mathrm{CD}^{+} \mathrm{T}$ cells in septic shock patients relative to HD (Supplementary Figure 2A-2C). However, no difference of TIGIT on CD45RA ${ }^{+}$effector memory ( $\mathrm{T}_{\mathrm{EMRA}}, \mathrm{CD} 45 \mathrm{RA}^{+} \mathrm{CCR}^{-}$) $\mathrm{CD}^{+}{ }^{+} \mathrm{T}$ cells was observed among three groups (Supplementary Figure 2D). With regard to the $\mathrm{CD}^{+}$compartment, the percentage of TIGIT on naïve, effector memory and central memory $\mathrm{CD}^{+} \mathrm{T}$ was significantly higher in septic shock group relative to HD 
(Supplementary Figure 2E-2G). Similarly, there was no difference in the TIGIT expression on $\mathrm{T}_{\text {EMRA }} \mathrm{CD} 8^{+} \mathrm{T}$ cells among HD, septic and septic shock patients (Supplementary Figure 2H).

\section{Elevated TIGIT is positively correlates with illness severity and inflammatory responses during sepsis}

To explore the association of TIGIT on T cells and the severity of organ injury, we found that the percentage of TIGIT on either $\mathrm{CD} 4^{+}$or $\mathrm{CD} 8^{+} \mathrm{T}$ cells was higher in patients with $\mathrm{SOFA} \geq 8$ than those with SOFA $<8$ (CD4: $p=0.013$, Figure 2A; CD8: $p=0.044$, Figure 2B). It has been reported that elevated TIGIT expression inhibits $\mathrm{T}$ cell immunity, and results in an inability to eradicate the primary infection and inducing secondary nosocomial infection (19). Therefore, we examined the relationship between TIGIT expression and nosocomial infection. Though there was no difference in TIGIT expression on bulk CD4 between non-nosocomial and nosocomial infection ( $p=0.6$, Figure 2C), the frequency of TIGIT on Foxp $3^{+} \mathrm{CD}^{+}$Treg was significantly higher in the patients with nosocomial infection ( $p=0.0021$, Figure 2D).

In order to explore the TIGIT expression and inflammation, we inquired about the correlation of TIGIT on T cells with inflammatory factors. Procalcitonin (PCT) has been considered a crucial acute-phase protein in response to bacterial infection, and positive correlations between PCT and the percentage of TIGIT $^{+}$Treg cells were identified (Figure 2E). Nonetheless, there was no relationship between TIGIT expression and C-reactive protein (data not shown). Results indicated that the frequencies of $\mathrm{TIGIT}^{+}$Treg cells positively correlated with systemic anti-inflammatory IL-10 levels during the first 24h of sepsis (Figure 2F), and had no relationship with other cytokines, including TNF, IFN- $\gamma$, IL-6, IL-2, IL-4, and IL-17 (data not shown). Meanwhile, TIGIT ${ }^{+}$Tconv cells significantly positively correlated with systemic pro-inflammatory IL-6 in the early stage of sepsis (Figure 2G). We also explored the correlation of TIGIT expression and other prognostic factors and found that the frequencies of TIGIT $^{+}$Treg cells positively correlated with ventilator time (Figure $2 \mathrm{H}$ ). Nonetheless, $\mathrm{TIGIT}^{+} \mathrm{T}$ cells had no relationship with $\mathrm{CD}^{+}$or $\mathrm{CD}^{+} \mathrm{T}$ cell absolute counts (data not shown). Not any other significant correlations of $\mathrm{TIGIT}^{+} \mathrm{T}$ cells with clinical parameters were observed.

\section{TIGIT $^{+}$T cells exhibits increased PD-1 and decreased CD226 expression during sepsis}


Multiple studies have shown that most of TIGIT expressing T cells co-expressed PD-1 on T cells $(14,20)$. Consistent with the previous study, we observed that $\mathrm{TIGIT}^{+} \mathrm{CD} 4^{+} \mathrm{T}$ cells expressed significantly higher PD- 1 than TIGIT-CD $4^{+} \mathrm{T}$ cells from PBMCs of septic patients $(p<0.0001$, Figure 3A-3B). Following similar pattern, $\mathrm{TIGIT}^{+} \mathrm{CD}^{+} \mathrm{T}$ cells exhibited increased expression of PD-1 relative to the TIGIT ${ }^{-}$compartment during sepsis $(p=0.009$, Figure $3 \mathrm{C})$.

TIGIT competes with costimulatory receptor CD226 on T cells. Thus we assessed the CD226 expression on $\mathrm{T}$ cells during sepsis (Figure 3D). The percentage of $\mathrm{CD} 226^{+}$on $\mathrm{TIGIT}^{+} \mathrm{CD} 4^{+} \mathrm{T}$ cells was downregulated compared with those on TIGIT ${ }^{-}$compartment during sepsis $(p=0.03$, Figure 3E). Likewise, the expression of $\mathrm{CD} 226^{+}$on $\mathrm{TIGIT}^{+} \mathrm{CD} 8^{+} \mathrm{T}$ cells was decreased relative to TIGIT $^{-}$compartments during sepsis $(p=0.03$, Figure $3 \mathrm{~F}$ ).

\section{TIGIT expressing $\mathbf{T}$ cells have impaired cytokine responses}

To explore the effects of TIGIT on T cell function, we evaluated the frequencies of cytokineproducing T cells. Intracellular cytokine staining following ex vivo re-stimulation revealed that TIGIT $^{+} \mathrm{CD}^{+}{ }^{+} \mathrm{T}$ cells produced significantly less TNF, IFN- $\gamma$, and IL-2 relative to TIGIT-CD4 ${ }^{+} \mathrm{T}$ cells in sepsis (Figure 4A-4D). For the $\mathrm{CD} 8^{+} \mathrm{T}$ cell compartment, $\mathrm{TIGIT}^{+} \mathrm{CD} 8^{+} \mathrm{T}$ cells produced less TNF, IFN- $\gamma$, and IL-2 relative to TIGIT-CD ${ }^{+} \mathrm{T}$ cells during sepsis (Figure 4E-4H). These data, including PD-1 and cytokine production, suggested that TIGIT $^{+} \mathrm{T}$ cells exhibited distinguishing features of exhausted $\mathrm{T}$ cells.

\section{Blockade of TIGIT reverses the frequencies of cytokine-producing $T$ cells}

Since the blockade of TIGIT limits virus infection and elicits anti-tumor responses (21), we evaluated the effects of TIGIT blockade on T cells using PBMCs from septic patients at $24 \mathrm{~h}$ after diagnosis of sepsis (Figure 5A). To better evaluate the ex vivo effect of anti-TIGIT antibody on the function of $\mathrm{T}$ cells, we incubated the PBMCs of septic patients with anti-TIGIT or isotype antibody for three days, followed by ex vivo re-stimulation with PMA and ionomycin. The blockade of TIGIT significantly increased the frequencies of TNF-producing $\mathrm{CD} 4^{+}$and $\mathrm{CD} 8^{+} \mathrm{T}$ cells (Figure 5B and 5C). Further, while anti-TIGIT Ab had no impact on the frequencies of IFN$\gamma$-producing cells among $\mathrm{CD} 4^{+} \mathrm{T}$ cells, it significantly increased IFN- $\gamma$ production by $\mathrm{CD} 8^{+} \mathrm{T}$ cells 
(Figure 5D and 5E). Anti-TIGIT Ab had a trend in the increase in IL-2 production by $\mathrm{CD}^{+} \mathrm{T}$ cells (Figure 5F), though it had no effect on the IL-2-producing $\mathrm{CD} 8^{+} \mathrm{T}$ cells during sepsis (Figure 5G).

\section{Discussion}

In this report, we profiled TIGIT expression on T cells and NK cells and found that TIGIT on T cells was significantly upregulated during sepsis, especially in the patients with septic shock, though sepsis had no impact on the TIGIT expression on NK cells. We unveiled that the percentage of TIGIT $^{+} \mathrm{T}$ cells positively correlated with inflammatory responses and ventilator time during sepsis progress. Further, the result demonstrated that the high expression of TIGIT on T cells increased PD-1 and decreased cytokine-producing T cells, which contributed to T cell exhaustion. The blockade of TIGIT led to the restoration of $\mathrm{T}$ cell exhaustion by increasing cytokine production.

The high mortality of sepsis has historically been attributed to unbridled cytokine-mediated inflammation during the early stage of sepsis. However, mounting evidence shows that hyperinflammation and immunosuppression occur simultaneously during sepsis $(22,23)$. Of note, septic patients with a protracted course and poor prognosis are mostly due to impaired host immunity, resulting in failure to control invading pathogens. Numerous mechanisms underlie sepsis-induced immunosuppression, including effector lymphocytes apoptosis, increased regulatory $\mathrm{T}$ cells and myeloid-derived suppressor cells, and a shift for Th1 to Th2 type (24-26). Among these, T cell exhaustion plays a crucial role in the immunosuppression during sepsis. $\mathrm{T}$ cell exhaustion is defined by increased expression of inhibitory checkpoint receptors, decreased ability of immune effector cells to produce cytokines, and failure to develop functional $\mathrm{T}$ cell memory (7). Overexpression of immune checkpoint inhibitors suggests a feature of sepsis-related immunosuppression and higher morality in sepsis (27). A recent clinical study showed that LAG3 and PD-1 had unique expression characteristics and possessed a synergistic effect on T cell exhaustion during sepsis (28). Though PD-1 is considered to be one of the most promising targets, it has been demonstrated that anti-PD-1 treatment did not fully reverse $T$ cell exhaustion in sepsis. Further study showed that PD- $1^{+} \mathrm{T}$ cells exhibited increased other coinhibitory molecules and decreased responding capacity to PD-1 blockade therapy due to the downregulation of 
costimulatory molecule CD28 during sepsis (20). Since different coinhibitory receptors expressed on exhausted $\mathrm{T}$ cells have different roles, novel targets to reverse $\mathrm{T}$ cell exhaustion is still needed to explore in the treatment of sepsis.

TIGIT has emerged as a crucial checkpoint receptor since its role was explored in anti-viral and anti-tumor responses $(16,21)$. It has been reported that TIGIT expressions on T and NK cells were upregulated upon antigen exposure in HIV-infected and tumor individuals $(14,29)$. Sepsis is an immunopathological process with persistent antigens exposure in the host response to pathogens. In the study, we firstly reported TIGIT expression on T cells and NK cells in the sepsis and septic shock patients. The result indicated that the levels of TIGIT on $\mathrm{CD} 4^{+}$and $\mathrm{CD} 8^{+} \mathrm{T}$ cells were both upregulated in the septic patients. Strikingly, with the increasing severity of sepsis, TIGIT on T cells increased more significantly in septic shock patients than non-shock septic individuals. Furthermore, elevated TIGIT is positively related to increasing levels of inflammatory cytokines and ventilator time. It suggested that $\mathrm{TIGIT}^{+} \mathrm{T}$ cells could be an important indicator for immunity detection and prognostic evaluation.

Several studies on humans and animals indicated that TIGIT negatively modulates T and NK cell function (30-32). Signaling through TIGIT/CD155 with the recruitment of phosphatases can curtail $\mathrm{T}$ cell and $\mathrm{NK}$ cell function. An initial study demonstrated that TIGIT exerted immunosuppressive by triggering CD155 in dendritic cells (DCs), thereby preventing DCs maturation and inducing IL-10 production (13). Another study showed that the expression of TIGIT inhibited NK cell function in the HIV model (29). Our data exhibited that the high expression of TIGIT induced increased PD-1 and decreased CD226 expression, a competing costimulator receptor on $\mathrm{T}$ cells in sepsis. It has been demonstrated that the upregulation of PD-1 pathway is associated with higher mortality (27). Since TIGIT has a much higher affinity than CD226 for binding with CD155, the effects of CD226 on activating T cells could be repressed by overexpression of TIGIT during sepsis. 
Recently, immune checkpoint blockade therapies have garnered attention in clinical research (33). Anti-TIGIT only or in combination with PD-1 blockade has been emerging to a promising therapy in anti-tumor treatment $(16,34)$. Our data show that blocking TIGIT signaling with anti-TIGIT antibody reserves the function of $\mathrm{T}$ cells from PBMCs extracted from septic patients. The combination of the blockade of TIGIT and PD-1 warrants further study in septic patients. Furthermore, due to the complex correlation of immune inhibitory receptors, deeper investigations into the conditions under which TIGIT blockade impacts other coinhibitory molecule expression and signaling are required.

There are still some limitations in our study. First, the study enrolled a limited sample size and was conducted in s single center. The findings need to be confirmed in a large multi-center study. Second, healthy donors were used as controls instead of non-sepsis critical illness controls since

critically ill patients exhibit many immune deficits that could be distinguished from septic patients. Third, we only observed the early stage of sepsis, and further study on the late stage of sepsis was still warranted. Finally, the study was an observational study and further investigations into interfering with TIGIT signaling during sepsis are required.

\section{Conclusions}

In the current study, we first report that the TIGIT expression on $\mathrm{T}$ cells is upregulated in the patients with septic shock and confirms the crucial role of TIGIT in T cells exhaustion during sepsis. Furthermore, the increasing TIGIT $^{+} \mathrm{T}$ cells are associated with inflammatory responses and poor outcome of sepsis. Of note, while blockade of TIGIT can reverse T cell exhaustion, it suggests that reversal of $\mathrm{T}$ cell exhaustion using anti-TIGIT offers a potential novel immune therapeutic strategy for treating sepsis.

\section{List of Abbreviations}

APACHE II: Acute Physiology and Chronic Health Evaluation II; CTLA-4: Cytotoxic T lymphocyte antigen-4; CRP: C-reactive protein; FMO: Fluorescence minus one; HD: Healthy 
donors; IFN- $\gamma$ : Interferon-gamma; IL: interleukin; LAG-3: Lymphocyte-activation gene 3; NK: Natural killer cells; PD-1: Programmed cell death 1; PD-L1: Programmed cell death ligand-1; PCT: procalcitonin; PBMCs: Peripheral blood mononuclear cells; SOFA: Sequential organ failure assessment; TIM-3: T cell immunoglobin mucin-3; TIGIT: T cell immunoglobin and ITIM domain; TNF: Tumor necrosis factor; Treg: Regulatory T cells; Tconv: Conventional T cells; WBC: White blood cell count.

\section{Declarations}

\section{Ethics Approval and Consent to Participate}

The Medical Science Research Ethics Committee of the First Affiliated Hospital of China Medical University (Shenyang, China) approved the study protocol ([2015]2015-113-2). Our study was conducted according to the principles of the Declaration of Helsinki. Written informed consent was obtained from all the patients included and healthy donors.

\section{Consent for publication}

Not applicable

\section{Availability of data and material}

All data generated or analysed during this study are included in this published article and its supplementary information files.

\section{Authors' Contributions}

YS and XM designed research studies; YS, YC, JL conducted experiments; YS and RD analysed the data; RD, YC and JL helped to recruit study participants; XM and YS wrote the manuscript. All authors read and approved the final manuscript.

\section{Funding}

The authors would like to acknowledge support from the project of the Science and Technology of Liaoning Province (2020. JH2/10300010).

\section{Competing Interests}

The authors declare that they have no competing interests. 


\section{Acknowledgments}

We thank the Science and Technology of Liaoning Province for providing platform and funding for the project. We appreciate Dr. Zining Zhang from the Key Laboratory of AIDS Immunology of Liaoning Province for her consultation in flow analysis and helpful discussion. We would like to extend a profound appreciation to each healthy donor in the study, including my dear colleagues.

\section{REFERENCES}

1.Singer M, Deutschman CS, Seymour CW, Shankar-Hari M, Annane D, Bauer M, et al. The Third International Consensus Definitions for Sepsis and Septic Shock (Sepsis-3). Jama. 2016;315(8):801-10.

2.Hotchkiss RS, Monneret G, Payen D. Sepsis-induced immunosuppression: from cellular dysfunctions to immunotherapy. Nat Rev Immunol. 2013;13(12):862-74.

3. Scicluna BP, van Vught LA, Zwinderman AH, Wiewel MA, Davenport EE, Burnham KL, et al. Classification of patients with sepsis according to blood genomic endotype: a prospective cohort study. Lancet Respir Med. 2017;5(10):816-26.

4.van der Poll T, van de Veerdonk FL, Scicluna BP, MG. N. The immunopathology of sepsis and potential therapeutic targets. Nat Rev Immunol. 2017;17(7):407-20.

5.Boomer JS, To K, Chang KC, Takasu O, Osborne DF, Walton AH, et al. Immunosuppression in patients who die of sepsis and multiple organ failure. Jama. 2011;306(23):2594-605.

6.Jonathan S Boomer, Jennifer Shuherk-Shaffer, Richard S Hotchkiss, Green JM. A prospective analysis of lymphocyte phenotype and function over the course of acute sepsis. Crit Care. 2012;16:R112.

7.Katherine Chang, Catherine Svabek, Cristina Vazquez-Guillamet, Bryan Sato, David Rasche, Strother Wilson, et al. Targeting the programmed cell death 1: programmed cell death ligand 1 pathway reverses T cell exhaustion in patients with sepsis. Crit Care. 2014;18:R3.

8.Hotchkiss RS, Colston E, Yende S, Crouser ED, Martin GS, Albertson T, et al. Immune checkpoint inhibition in sepsis: a Phase $1 \mathrm{~b}$ randomized study to evaluate the safety, tolerability, pharmacokinetics, and pharmacodynamics of nivolumab. Intensive care medicine. 2019;45(10):1360-71.

9.Chang KC, Burnham CA, Compton SM, Rasche DP, Mazuski RJ, McDonough JS, et al. Blockade of the negative co-stimulatory molecules PD-1 and CTLA-4 improves survival in primary and secondary fungal sepsis. Crit Care. 2013;17(3):R85.

10.Day CL, Kaufmann DE, Kiepiela P, Brown JA, Moodley ES, Reddy S, et al. PD-1 expression on HIV-spe- cific T cells is associated with T-cell exhaustion and disease progression. Nature. 2006;443:350-4. 
11.Yamamoto T, Price DA, Casazza JP, Ferrari G, Nason M, Chattopadhyay PK, et al. Surface expression patterns of negative regulatory molecules identify determinants of virus-specific CD8+ T-cell exhaustion in HIV infection. Blood. 2011;117:4805-15.

12.Anderson AC, Joller N, Kuchroo VK. Lag-3, Tim-3, and TIGIT: Co-inhibitory Receptors with Specialized Functions in Immune Regulation. Immunity. 2016;44(5):989-1004.

13. Yu X, Harden K, Gonzalez LC, Francesco M, Chiang E, Irving B, et al. The surface protein TIGIT suppresses $\mathrm{T}$ cell activation by promoting the generation of mature immunoregulatory dendritic cells. Nature immunology. 2009;10(1):48-57.

14.Chew GM, Fujita T, Webb GM, Burwitz BJ, Wu HL, Reed JS, et al. TIGIT Marks Exhausted T Cells, Correlates with Disease Progression, and Serves as a Target for Immune Restoration in HIV and SIV Infection. PLoS pathogens. 2016;12(1):e1005349.

15.Kong Y, Zhu L, Schell TD, Zhang J, Claxton DF, Ehmann WC, et al. T-Cell Immunoglobulin and ITIM Domain (TIGIT) Associates with CD8+ T-Cell Exhaustion and Poor Clinical Outcome in AML Patients. Clinical cancer research : an official journal of the American Association for Cancer Research. 2016;22(12):3057-66.

16.Johnston RJ, Comps-Agrar L, Hackney J, Yu X, Huseni M, Yang Y, et al. The immunoreceptor TIGIT regulates antitumor and antiviral $\mathrm{CD} 8(+) \mathrm{T}$ cell effector function. Cancer cell. 2014;26(6):923-37.

17.Meng Y, Zhao ZZ, Zhu WZ, Yang T, Deng XM, R B. CD155 blockade improves survival in experimental sepsis by reversing dendritic cell dysfunction. Biochemical and Biophysical Research Communications. 2017;490:283-9.

18.Deng CW, Li WL, Fei YY, Wang L, Chen YY, Zeng XF, et al. Imbalance of the CD226/TIGIT Immune Checkpoint Is Involved in the Pathogenesis of Primary Biliary Cholangitis. Front Immunol. 2020;11:1619.

19.Zhang C, Lin RY, Li ZD, Yang S, Bi XJ, Wang H, et al. Immune Exhaustion of T Cells in Alveolar Echinococcosis Patients and Its Reversal by Blocking Checkpoint Receptor TIGIT in a Murine Model. Hepatology. 2020;71(4):1297-315.

20.Chen CW, Xue M, Zhang WX, Xie JF, Coopersmith CM, Ford ML. 2B4 but not PD-1 blockade improves mortality in septic animals with preexisting malignancy. JCI Insight. 2019;4(22):e127867.

21.Chauvin JM, Pagliano O, Fourcade J, Sun Z, Wang H, Sander C, et al. TIGIT and PD-1 impair tumor anti- gen-specific CD8+ T cells in melanoma patients. The Journal of clinical investigation. 2015;125(5):2046-58.

22.Xiao W, Mindrinos MN, Seok J, Cuschieri J, Cuenca AG, Gao H, et al. A genomic storm in critically injured humans. J Exp Med. 2011;208:2581-90.

23. Shigeaki Inoue, Kodai Suzuki, Yukako Komori, Yukiko Morishita, Kyoko Suzuki-Utsunomiya, Katsuto Hozumi, et al. Persistent inflammation and T cell exhaustion in severe sepsis in the elderly. Crit Care. 2014;18:R130.

24.Munford RS, J P. Normal responses to injury prevent systemic inflammation and can be immunosuppressive. Am J Respir Crit Care Med. 2001;163:316-21.

25.Venet F, Chung CS, Monneret G, Huang X, Horner B, Garber M, et al. Regulatory T cell populations in sepsis and trauma. Journal of leukocyte biology. 2008;83:523-35.

26.Delano MJ, Scumpia PO, Weinstein JS, Coco D, Nagaraj S, Kelly-Scumpia KM, et al. MyD88dependent expansion of an immature GR-1(+)CD11b(+) population induces $\mathrm{T}$ cell suppression and Th2 polarization in sepsis. J Exp Med. 2007;204:1463-74. 
27.Wilson JK, Zhao Y, Singer M, Spencer J, Shankar-Hari M. Lymphocyte subset expression and serum concentrations of PD-1/PD-L1 in sepsis - pilot study. Crit Care. 2018;22:95.

28.Niu B, Zhou F, Su Y, Wang L, Xu Y, Yi Z, et al. Different Expression Characteristics of LAG3 and PD-1 in Sepsis and Their Synergistic Effect on T Cell Exhaustion: A New Strategy for Immune Checkpoint Blockade. Front Immunol. 2019;10:1888.

29. Yin X, Liu T, Wang Z, Ma M, Lei J, Zhang Z, et al. Expression of the Inhibitory Receptor TIGIT Is Up-Regulated Specifically on NK Cells With CD226 Activating Receptor From HIVInfected Individuals. Front Immunol. 2018;9:2341.

30.Joller N, Hafler JP, Brynedal B, Kassam N, Spoerl S, Levin SD, et al. Cutting edge: TIGIT has T cell-intrinsic inhibitory functions. Journal of immunology. 2011;186(3):1338-42.

31.Joller N, Lozano E, Burkett PR, Patel B, Xiao S, Zhu C, et al. Treg cells expressing the coinhibitory molecule TIGIT selectively inhibit proinflammatory Th1 and Th17 cell responses. Immunity. 2014;40(4):569-81.

32.Lozano E, Dominguez-Villar M, Kuchroo V HD. The TIGIT/CD226 axis regulates human T cell function. Journal of immunology. 2012;188:3869-75.

33. Watanabe E, Thampy LK, Hotchkiss RS. Immunoadjuvant therapy in sepsis: novel strategies for immunosuppressive sepsis coming down the pike. Acute medicine \& surgery. 2018;5(4):30915.

34.Kurtulus S, Sakuishi K, Ngiow SF, Joller N, Tan DJ, Teng MW, et al. TIGIT predominantly regulates the immune response via regulatory $\mathrm{T}$ cells. The Journal of clinical investigation. 2015;125(11):4053-62.

\section{Figure Legends}

Figure 1. Expression of TIGIT on $T$ cells is higher in septic patients relative to healthy controls. (A) Representative flow plots showing TIGIT expression on $\mathrm{CD}^{+}$and $\mathrm{CD}^{+} \mathrm{T}$ cells compared to FMO control among HD, non-shock septic and septic shock patients. (B) Summary data of comparison of the percentage of TIGIT expression on $\mathrm{CD}^{+} \mathrm{T}$ cells among HD ( $\left.\mathrm{n}=17\right)$, non-shock sepsis $(n=10)$ and septic shock $(n=15)$ groups. (C-D) Comparison of the percentage of TIGIT on Foxp $3^{+} \mathrm{CD}^{+}$Treg and Foxp3-CD4 ${ }^{+}$Tconv cells among three groups. (E) Graphs showed compiled data of TIGIT expression on $\mathrm{CD}^{+} \mathrm{T}$ cells among three groups. (F) Comparison of the percentage of TIGIT on NK cells among HD, non-shock septic and septic shock patients. Results were representative of four independent experiments. Groups were compared using one-way ANOVA analysis and Turkey's multiple comparisons test. ${ }^{* *}, p<0.01, * * *, p<0.0001$. 
Figure 2. Elevated TIGIT is positively correlated with illness severity and inflammatory responses during sepsis. (A-B) Summary data depicting TIGIT expression on bulk $\mathrm{CD}^{+}$and $\mathrm{CD}^{+} \mathrm{T}$ cells between SOFA $<8$ and SOFA $\geq 8$. (C-D) Summary data of comparison of the percentage of TIGIT on bulk $\mathrm{CD}^{+}$and Foxp $3^{+} \mathrm{CD} 4^{+}$Treg cells between non-nosocomial and nosocomial infection. (E) Analysis of the correlation between TIGIT expression on Treg cells and the levels of PCT. (F) Scatterplot of the correlation between TIGIT $^{+}$of Treg cells vs. serum IL-10. (G) Scatterplot of the correlation of TIGIT ${ }^{+}$of CD4 ${ }^{+}$Tconv cells vs. serum IL-6. (H) the correlation between the percentage of TIGIT of Treg and ventilator time. Each point in the scatter plot represented the value of two variables for a given observation. The Spearman's rank test was used for correlation analyses. * $, p<0.05, * *, p<0.01$.

Figure 3. TIGIT $^{+} \mathrm{T}$ cells exhibits higher PD-1 expression and lower CD226 expression in sepsis. (A) Representative flow plots showing the percentage of PD-1 on TIGIT $^{-}$vs. $^{\text {TIGIT }}{ }^{+} \mathrm{T}$ cells. (B-C) Graph shows compiled frequency (\%) of PD-1 from TIGIT $^{-}$vs. TIGIT $^{+} \mathrm{CD}^{+}$and $\mathrm{CD}^{+} \mathrm{T}$ cells in septic patients $(\mathrm{n}=18)$. (D) Representative flow plots showing the percentage of CD226 on TIGIT $^{-}$vs. TIGIT $^{+}$T cells from septic patients. (E-F) Summary data depicting frequency of CD226 from TIGIT $^{-}$vs. TIGIT $^{+} \mathrm{CD}^{+}$and $\mathrm{CD}^{+}$T cells in septic patients $(\mathrm{n}=6)$. P values were calculated using non-parametric Wilcoxon matched pairs test. *, $p<0.05$, **, $p<0.01$, ****, $p<0.0001$.

Figure 4. TIGIT expressing $T$ cells have impaired cytokine responses. Cryopreserved PBMCs were thawed and stimulated ex vivo with PMA and ionomycin for 5 hours, and were assessed for TNF, IFN- $\gamma$ and IL-2 production via intracellular cytokine staining. (A) Representative flow cytometry plots gated on $\mathrm{TIGIT}^{-}$vs. $\mathrm{TIGIT}^{+} \mathrm{CD}^{+} \mathrm{T}$ cells showing TNF, IFN- $\gamma$ and IL-2 secretion after no stimulation, stimulation with PMA and ionomycin. (B-D) Compiled data of TNF, IFN- $\gamma$ and IL-2 frequency (\%) from TIGIT $^{-}$vs. TIGIT $^{+} \mathrm{CD}^{+} \mathrm{T}$ cells compartments during sepsis $(\mathrm{n}=6)$. (E) Representative flow plots showed TNF, IFN- $\gamma$ and IL-2 secretion from TIGIT $^{-}$vs. TIGIT $^{+} \mathrm{CD}^{+} \mathrm{T}$ cells in sepsis. $(\mathrm{F}-\mathrm{H})$ Summary data depicting $\mathrm{CD} 8^{+} \mathrm{T}$ cell cytokine staining $(\mathrm{n}=6)$. $\mathrm{P}$ values were calculated by Wilcoxon matched pairs test. *,$p<0.05$. 
Figure 5. Blockade with anti-TIGIT reverses the frequencies of cytokine-producing $\mathbf{T}$ cells. Ex vivo PBMCs from septic patients were stimulated with PMA and ionomycin in the presence of isotype IgG or anti-TIGIT Ab. (A) Representative flow cytometry plots showed TNF-producing $\mathrm{CD}^{+}$and $\mathrm{CD}^{+} \mathrm{T}$ cells from septic patients with after no stimulation, treated with isotype IgG and anti-TIGIT Ab. (B-C) Summary of compiled data of the frequencies of TNF by the CD4 ${ }^{+}$and $\mathrm{CD}^{+} \mathrm{T}$ cells after treated with isotype IgG and anti-TIGIT Ab in septic patients ( $\mathrm{n}=7$ ). (D-E) Compiled data of IFN- $\gamma$ frequency (\%) after treatment with anti-TIGIT and isotype control during sepsis $(n=7)$. (F-G) Summary data depicting the percentage of PD-1 of CD4 ${ }^{+}$and $C D 8^{+} \mathrm{T}$ cells treated with isotype IgG or anti-TIGIT Ab $(n=7)$. P values were calculated by Wilcoxon matched pairs test. ${ }^{*}, p<0.05$. 


\section{Figures}

Figure 1

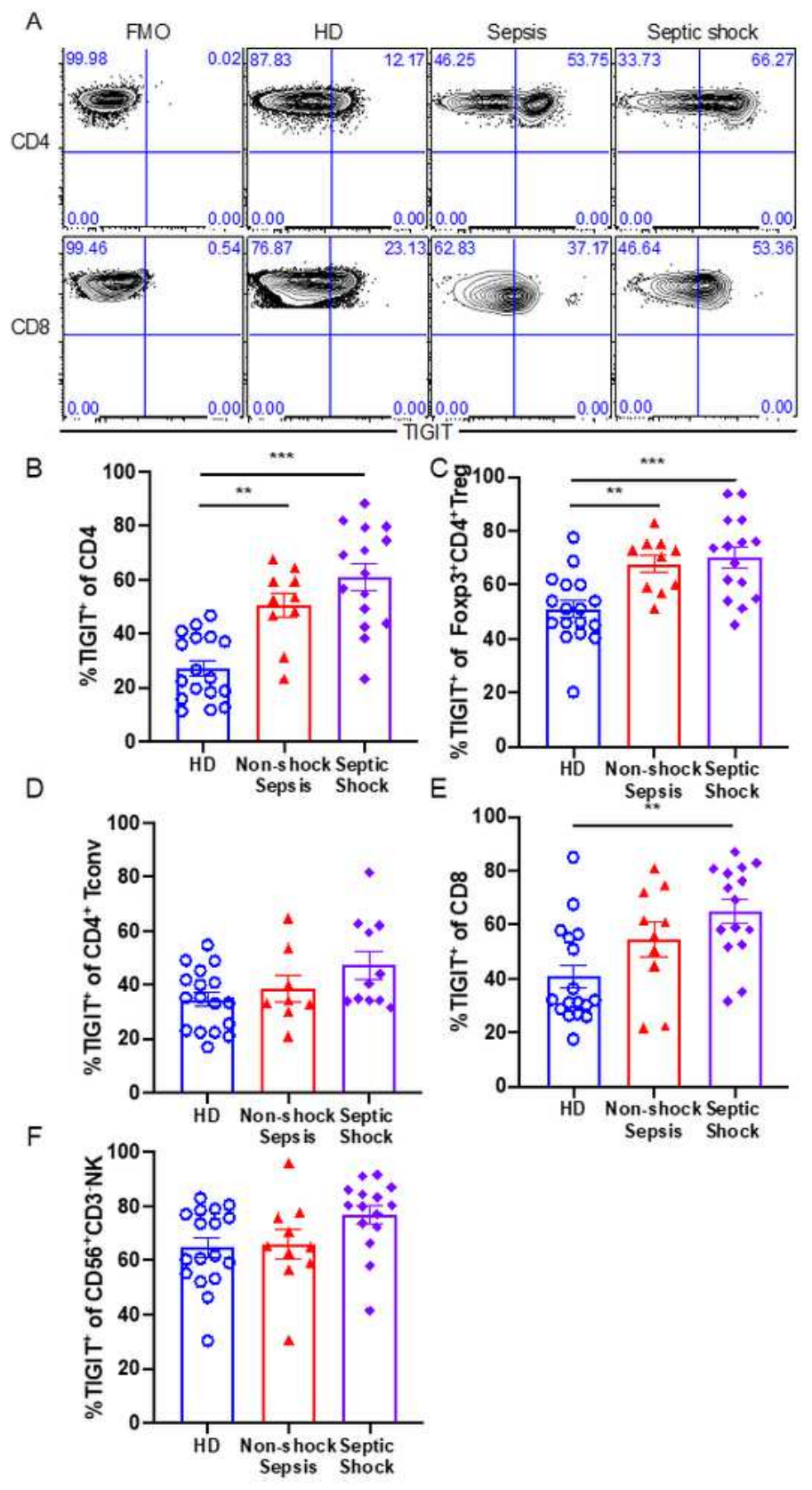

Figure 1

Expression of TIGIT on T cells is higher in septic patients relative to healthy controls. (A) Representative flow plots showing TIGIT expression on CD4+ and CD8+ T cells compared to FMO control among HD, non-shock septic and septic shock patients. (B) Summary data of comparison of the percentage of TIGIT 
expression on CD4+ T cells among HD ( $n=17)$, non-shock sepsis $(n=10)$ and septic shock $(n=15)$ groups. (C-D) Comparison of the percentage of TIGIT on Foxp3+CD4+ Treg and Foxp3-CD4+ Tconv cells among three groups. (E) Graphs showed compiled data of TIGIT expression on CD8+ T cells among three groups. (F) Comparison of the percentage of TIGIT on NK cells among HD, non-shock septic and septic shock patients. Results were representative of four independent experiments. Groups were compared using oneway ANOVA analysis and Turkey's multiple comparisons test. **, $p<0.01, * \star \star, p<0.0001$.

Figure 2
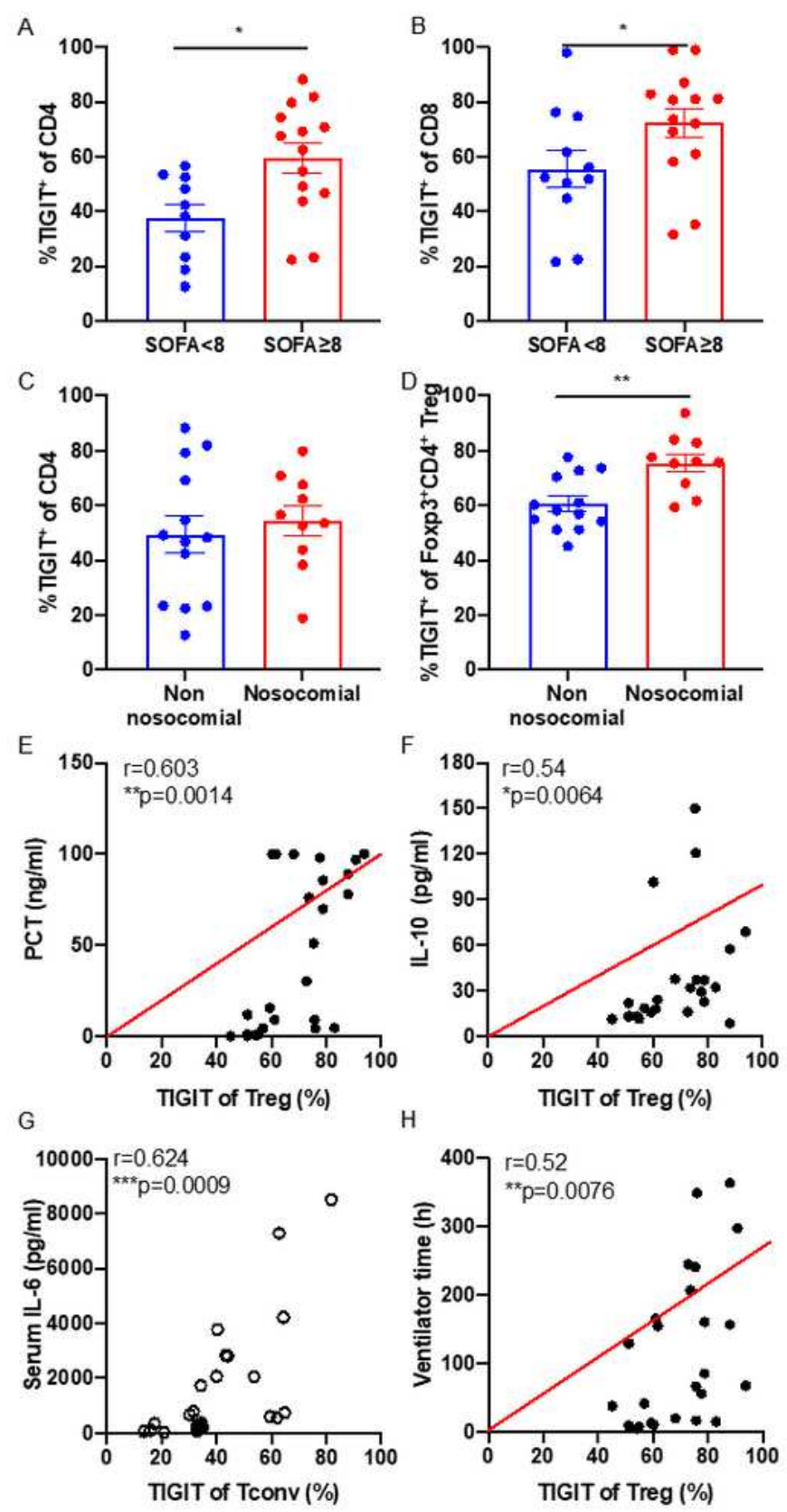

Figure 2 
Elevated TIGIT is positively correlated with illness severity and inflammatory responses during sepsis. (AB) Summary data depicting TIGIT expression on bulk CD4+ and CD8+ T cells between SOFA $<8$ and SOFA $\geq 8$. (C-D) Summary data of comparison of the percentage of TIGIT on bulk CD4+ and Foxp3+CD4+ Treg cells between non-nosocomial and nosocomial infection. (E) Analysis of the correlation between TIGIT expression on Treg cells and the levels of PCT. (F) Scatterplot of the correlation between TIGIT+ of Treg cells vs. serum IL-10. (G) Scatterplot of the correlation of TIGIT+ of CD4+ Tconv cells vs. serum IL-6. $\mathrm{H} H$ the correlation between the percentage of TIGIT of Treg and ventilator time. Each point in the scatter plot represented the value of two variables for a given observation. The Spearman's rank test was used for correlation analyses. ${ }^{*}, p<0.05, * \star, p<0.01$. 
Figure 3
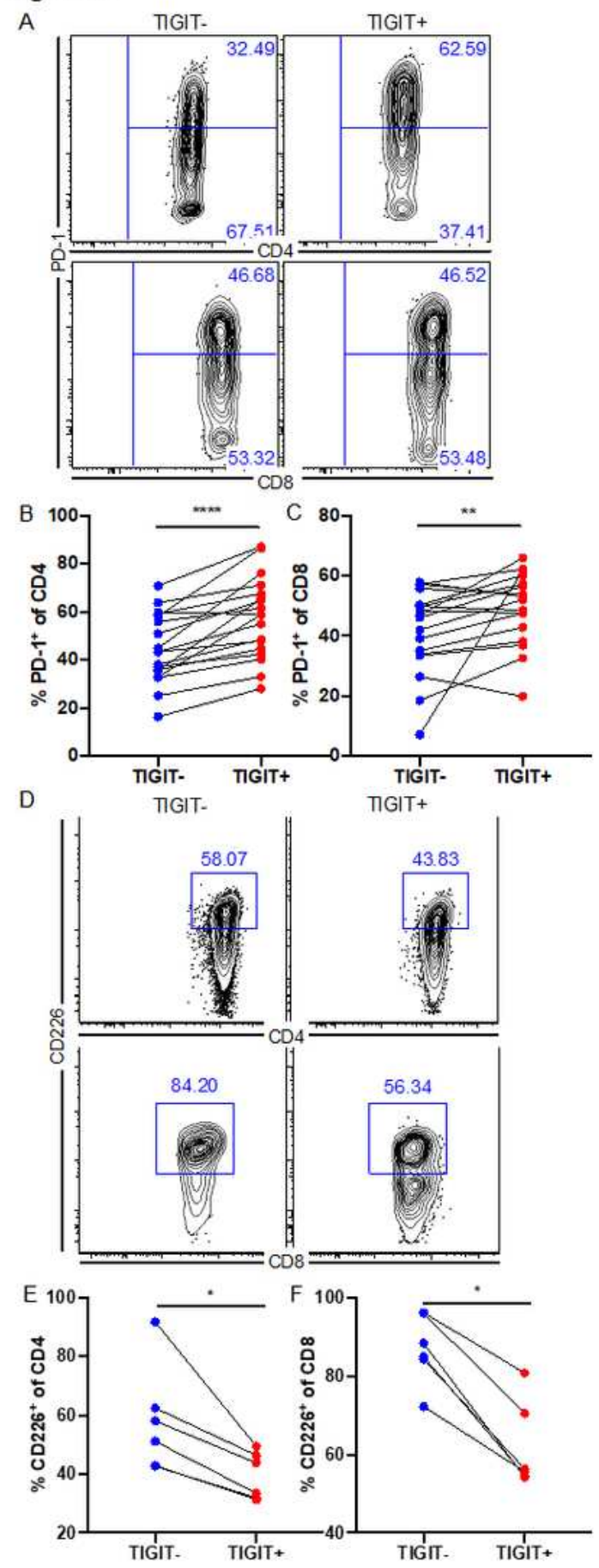

\section{Figure 3}

TIGIT+ T cells exhibits higher PD-1 expression and lower CD226 expression in sepsis. (A) Representative flow plots showing the percentage of PD-1 on TIGIT- vs. TIGIT+ T cells. (B-C) Graph shows compiled frequency (\%) of PD-1 from TIGIT- vs. TIGIT+CD4+ and CD8+ T cells in septic patients $(n=18)$. (D) Representative flow plots showing the percentage of CD226 on TIGIT- vs. TIGIT+ T cells from septic patients. (E-F) Summary data depicting frequency of CD226 from TIGIT- vs. TIGIT+CD4+ and CD8+ T 
cells in septic patients $(n=6)$. P values were calculated using non-parametric Wilcoxon matched pairs test. $\star, p<0.05, * \star, p<0.01, \star \star \star \star, p<0.0001$.

Figure 4

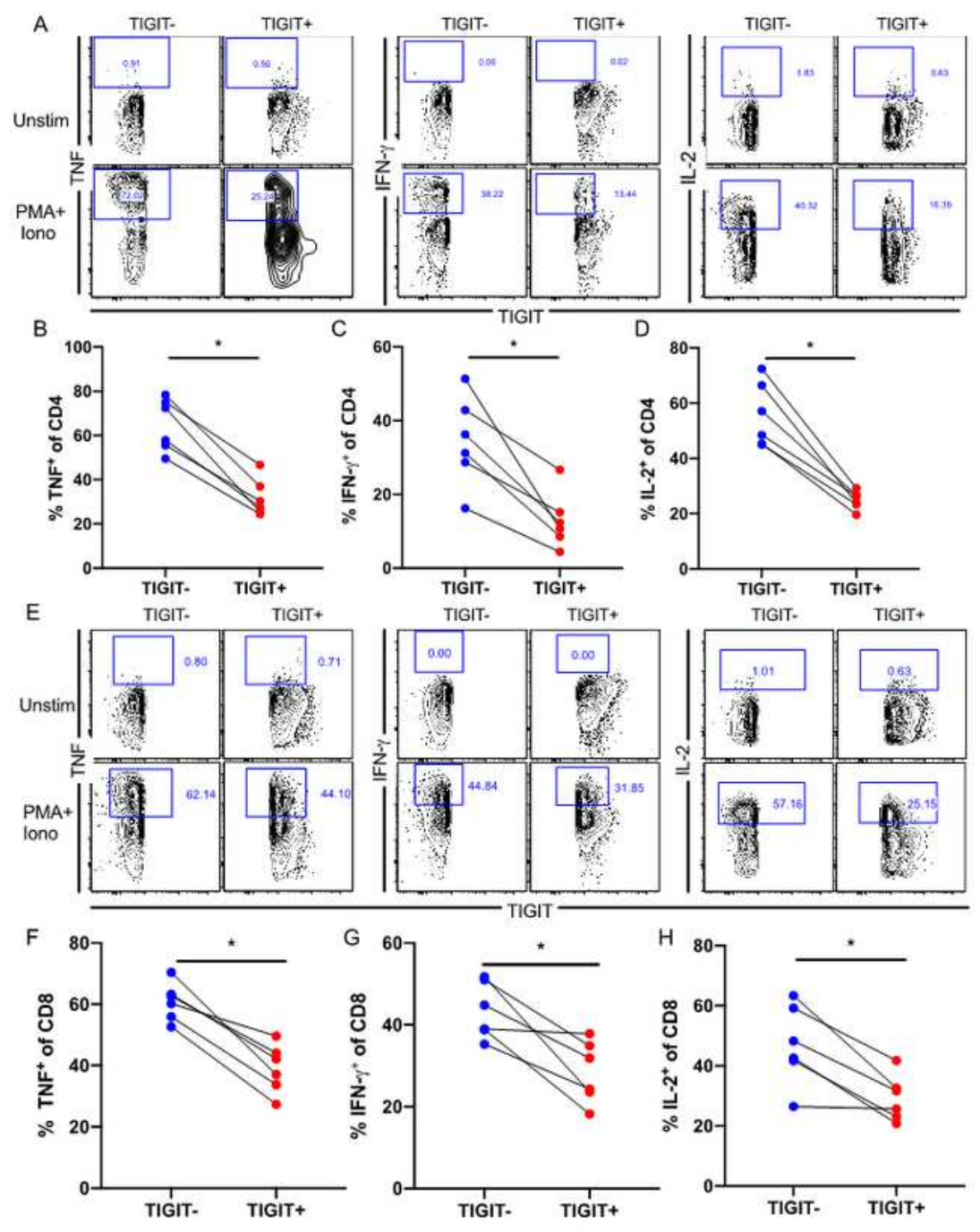

Figure 4

TIGIT expressing T cells have impaired cytokine responses. Cryopreserved PBMCs were thawed and stimulated ex vivo with PMA and ionomycin for 5 hours, and were assessed for TNF, IFN- $\gamma$ and IL-2 production via intracellular cytokine staining. (A) Representative flow cytometry plots gated on TIGIT- vs. 
TIGIT+CD4+ T cells showing TNF, IFN- $\gamma$ and IL-2 secretion after no stimulation, stimulation with PMA and ionomycin. (B-D) Compiled data of TNF, IFN-y and IL-2 frequency (\%) from TIGIT- vs. TIGIT+CD4+ T cells compartments during sepsis $(n=6)$. (E) Representative flow plots showed TNF, IFN-y and IL-2 secretion from TIGIT- vs. TIGIT+CD8+ T cells in sepsis. (F-H) Summary data depicting CD8+ T cell cytokine staining $(n=6)$. P values were calculated by Wilcoxon matched pairs test. *, $p<0.05$.

Figure 5

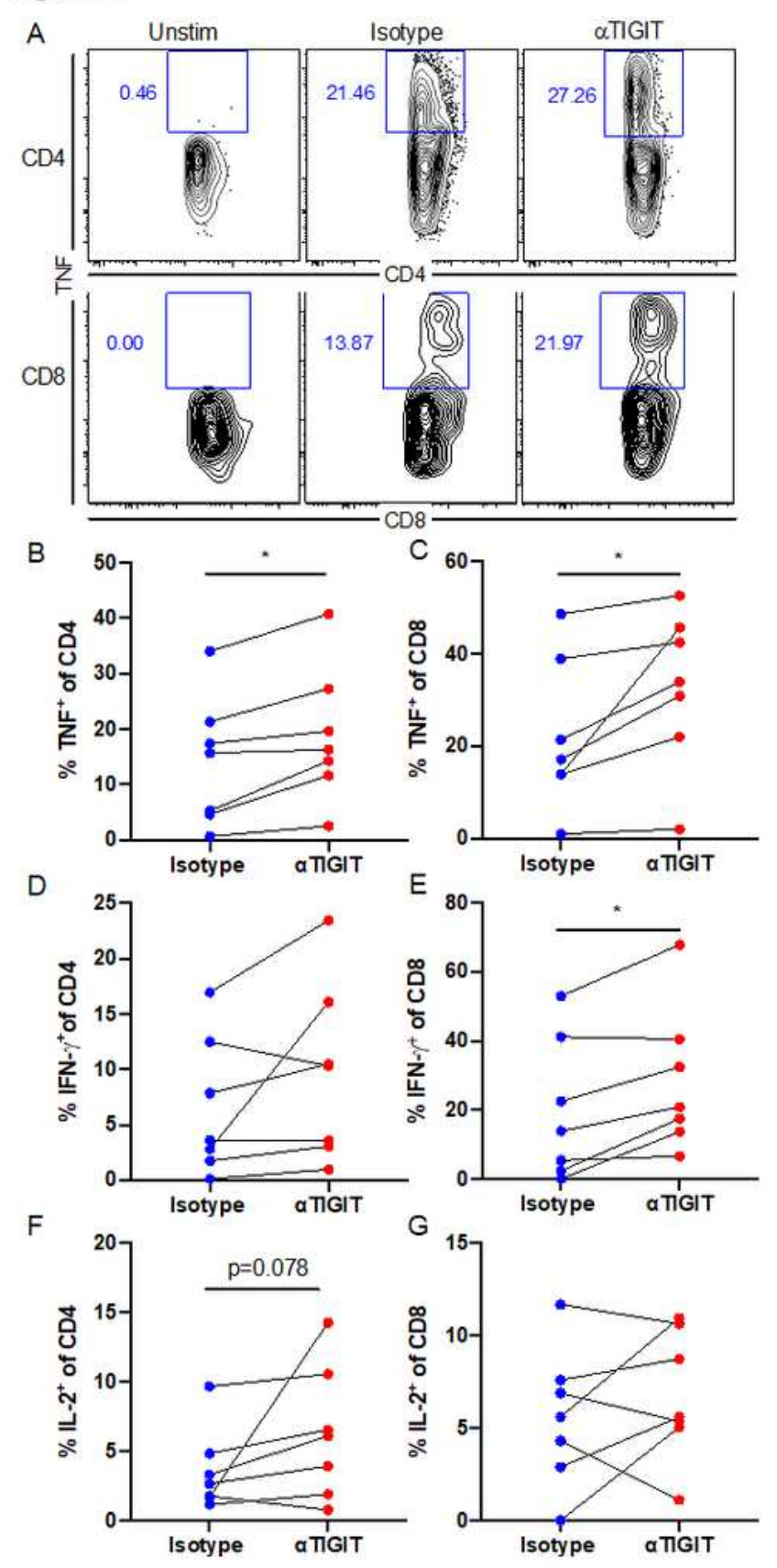

Figure 5 
Blockade with anti-TIGIT reverses the frequencies of cytokine-producing T cells. Ex vivo PBMCs from septic patients were stimulated with PMA and ionomycin in the presence of isotype IgG or anti-TIGIT Ab. (A) Representative flow cytometry plots showed TNF-producing CD4+ and CD8+ T cells from septic patients with after no stimulation, treated with isotype IgG and anti-TIGIT Ab. (B-C) Summary of compiled data of the frequencies of TNF by the CD4+ and CD8+ T cells after treated with isotype IgG and anti-TIGIT $A b$ in septic patients ( $n=7)$. (D-E) Compiled data of IFN-y frequency (\%) after treatment with anti-TIGIT and isotype control during sepsis $(n=7)$. ( $F-G)$ Summary data depicting the percentage of PD-1 of CD4+ and CD8+ T cells treated with isotype IgG or anti-TIGIT Ab $(n=7)$. P values were calculated by Wilcoxon matched pairs test. *, $p<0.05$.

\section{Supplementary Files}

This is a list of supplementary files associated with this preprint. Click to download.

- SupplementaryMaterial.docx 